\title{
Unidirectional all-fiber thulium-doped laser based on theta cavity and fiber Bragg grating as filtering element
}

\author{
Svyatoslav Kharitonov, Camille-Sophie Brès \\ Photonic Systems Laboratory, Ecole Polytechnique Fédérale de Lausanne (EPFL) STI IEL, CH-1015 Lausanne, Switzerland \\ svyatoslav.kharitonov@epfl.ch
}

\begin{abstract}
We present first all-fiber unidirectional ring thulium-doped laser, based on isolator-free theta cavity configuration and fiber Bragg mirror as filtering element. Laser provides $1 \mathrm{~W}$ output power with $30 \%$ slope efficiency, and linewidth of about $0.2 \mathrm{~nm}$.

OCIS codes: (060.3510) Lasers, fiber; (060.2390) Fiber optics, infrared; (160.5690) Rare-earth-doped materials
\end{abstract}

\section{Introduction}

Thulium-doped fiber lasers (TDFL), emitting the light in a broad wavelength range from approximately $1800 \mathrm{~nm}$ to $2100 \mathrm{~nm}$, gain a growing attention of research and industrial community, due to their numerous applications in spectroscopy, ranging, material and biological tissues processing as well as telecommunications [1]-[3]. Moreover, tunable narrow-linewidth lasers are used as pumping sources to drive nonlinear effects in optical fibers and waveguides, enabling a study of nonlinear processes at wavelengths that are not easily accessible with conventional semiconductor external cavity lasers [4], [5]. In most ring cavity TDFL implementations, grating-based filters [6], [7], Fabry-Pérot etalon [8], or combination of optical circulator with fiber Bragg grating (FBG) [5] are used as a wavelength selective element. While being packaged and interfaced with fiber pigtails, these filters remain inherently free-space optical components, which are sensitive to alignment fluctuations and have lower damage threshold, comparing to all-fiber elements. Additionally, optical isolator should be inserted into the cavity to ensure unidirectional lasing. The fibre isolator conventionally includes Faraday rotators and $45^{\circ}$ cross polarizers with adjacent free-space optics [9] and suppresses backward propagating light within a given bandwidth, generally not exceeding several tens of $\mathrm{nm}$. Therefore, isolator-free unidirectional ring fibre cavity (sometimes referred to "theta" or "yin-yang" resonators [10]) represents an attractive and cost-effective alternative solution. In theta cavities, nonreciprocal losses are introduced by providing an S-shape feedback within the main ring. We have already shown a core-pumped continuous wave TDFL that provides sub-Watt with a slope efficiency of $25 \%, 2 \mathrm{~dB}$ flat tuning range of 1900-2050 nm, and linewidth of $0.2 \mathrm{~nm}$, and achieves the extinction ratio (ER) of 18-25 dB between the favored and suppressed lasing directions [11]. The TDFL wavelength was tuned by fiberized grating filter. A high power Qswitched theta cavity TDFL using carbon nanotube saturable absorber was also reported [12].

In this paper, we present truly all-fiber narrow linewidth unidirectional ring TDFL, relying on theta cavity and FBG as wavelength selective element. The need for a circulator is circumvented by leveraging the already existing architecture. The laser output power reaches $1 \mathrm{~W}$, with 30\% slope efficiency and spectral width less than $0.22 \mathrm{~nm}$.

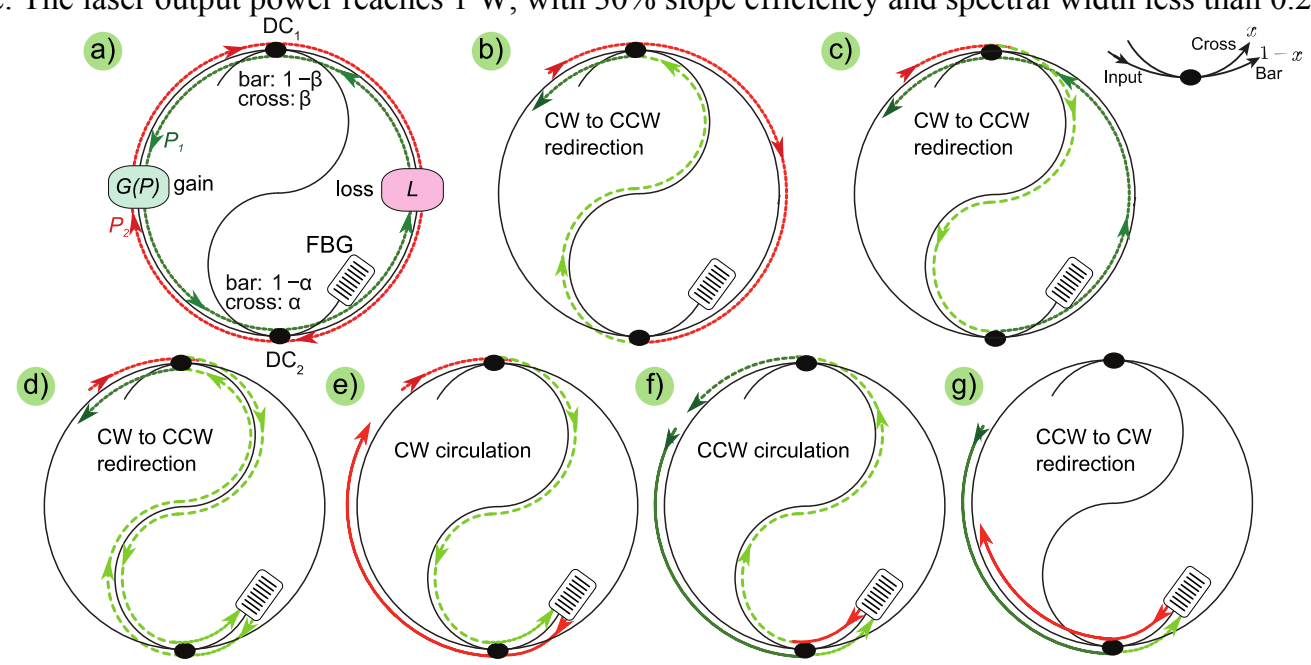

Fig. 1. Theta cavity with FBG layout. Optical path, not affected by FBG: a) Main paths for the clockwise (CW - red) and the counter-clockwise ( $\mathrm{CCW}$ - green) propagating modes, corresponding to a ring; $\mathrm{b}-\mathrm{c})$ two possible rectifying path, redirecting the $\mathrm{CW}$ modes towards the $\mathrm{CCW}$ modes. Optical path, influenced by FBG: d) $\mathrm{CW}$ to $\mathrm{CCW}$ redirection; e) $\mathrm{CW}$ circulation; f) $\mathrm{CCW}$ circulation; g) $\mathrm{CCW}$ to $\mathrm{CW}$ redirection. 


\section{Principle of operation}

The main idea behind theta resonator is a lasing direction rectification by introducing non-reciprocal cavity losses, described in details in [11]. We consider a ring resonator that consists of a lumped gain unit $G(P)$, and two directional couplers, which cross-outputs are connected together to form the S-shape feedback (Fig. 1). $P_{1}$ and $P_{2}$ are counter-clockwise $(\mathrm{CCW})$ and clockwise signals entering the amplifying unit. The unused port of the directional couplers can be cleverly exploited by connecting a FBG. If no FBG is present in the design, only three possible paths are sustained: $\mathrm{CCW}$ and $\mathrm{CW}$ circulation in the main ring (Fig. 1a), and two rectifying paths from $\mathrm{CW}$ to $\mathrm{CCW}$ modes (Fig. 1b-c). If a FBG is connected to the free port of directional coupler $2\left(\mathrm{DC}_{2}\right)$, one obtains four extra paths, involving the grating: $\mathrm{CW}$ to $\mathrm{CCW}$ redirection, $\mathrm{CW}$ circulation, $\mathrm{CCW}$ circulation, and $\mathrm{CCW}$ to $\mathrm{CW}$ redirection (Fig. 1d-g). Therefore, the laser spectral line shape can be controlled by the FBG in reflection mode, which provides a wavelength selective feedback in the cavity, and that without the need for a circulator or any modification to the original cavity.

\section{Fiber laser implementation and results}

The experimental setup of the theta cavity TDFL with FBG is shown in Fig. 2a. The gain unit (GU) consists of 11.5 $\mathrm{m}$ of thulium doped fibre (TmDF200, OFS Fitel Denmark ApS) bi-directionally core pumped with a $1600 \mathrm{~nm}$ pump obtained from an amplified tunable laser source (TLS). The S-shape feedback is formed by two identical directional couplers $\left(\mathrm{DC}_{1,2}\right)$, connected in such a manner that cross-coupling ratios are $90 \%$ and $10 \%$ for $\mathrm{DC}_{1}$ and $\mathrm{DC}_{2}$, respectively. Different FBGs were attached to a free port of $\mathrm{DC}_{2}$ : chirped $\mathrm{FBG}$ with central wavelength of $1980 \mathrm{~nm}$, and non-chirped FBGs at 2000, 2008, $2040 \mathrm{~nm}$. The third monitoring 5\% coupler $\left(\mathrm{DC}_{3}\right)$ is only included to evaluate the ER between CCW and CW modes. To extract the maximum possible power, a free port of $\mathrm{DC}_{1}$ is used as output.

a)

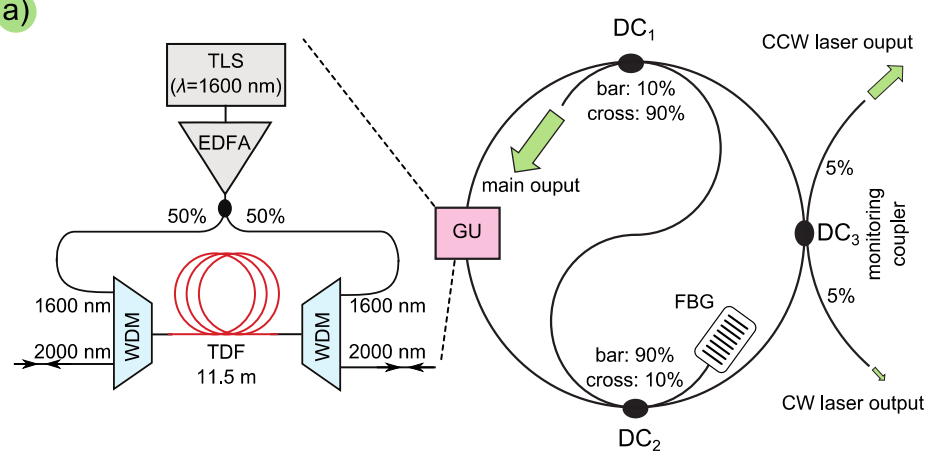

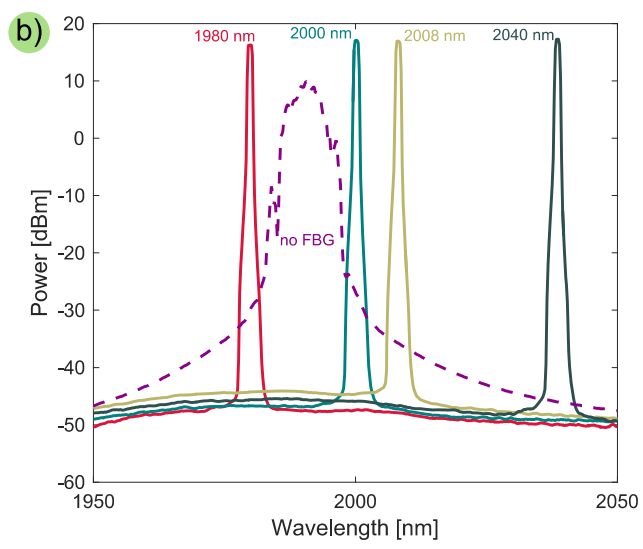

Fig. 2. Implementation of theta cavity TDFL. a) Experimental setup. Details of the gain unit (GU) are shown on the left. TLS: tunable laser source; EDFA: Erbium doped fibre amplifier; WDM: wavelength division multiplexer; TDF: Thulium doped fibre; FBG: fiber Bragg grating, DC: directional coupler. b) Attenuated emission spectra for different FBGs (pump power of $3 \mathrm{~W}, 1 \mathrm{~nm}$ resolution).

Without a FBG, the cavity lases around the emission peak at $1990 \mathrm{~nm}$, maintaining, however, unidirectional operation with $22 \mathrm{~dB}$ ER (Fig. 2b). Once a FBG is inserted, the TDFL generates a narrowband signal, tunable within entire emission bandwidth (Fig. $2 \mathrm{~b}$ - low resolution, Fig. 3 - high resolution), with ER better than $21 \mathrm{~dB}$.
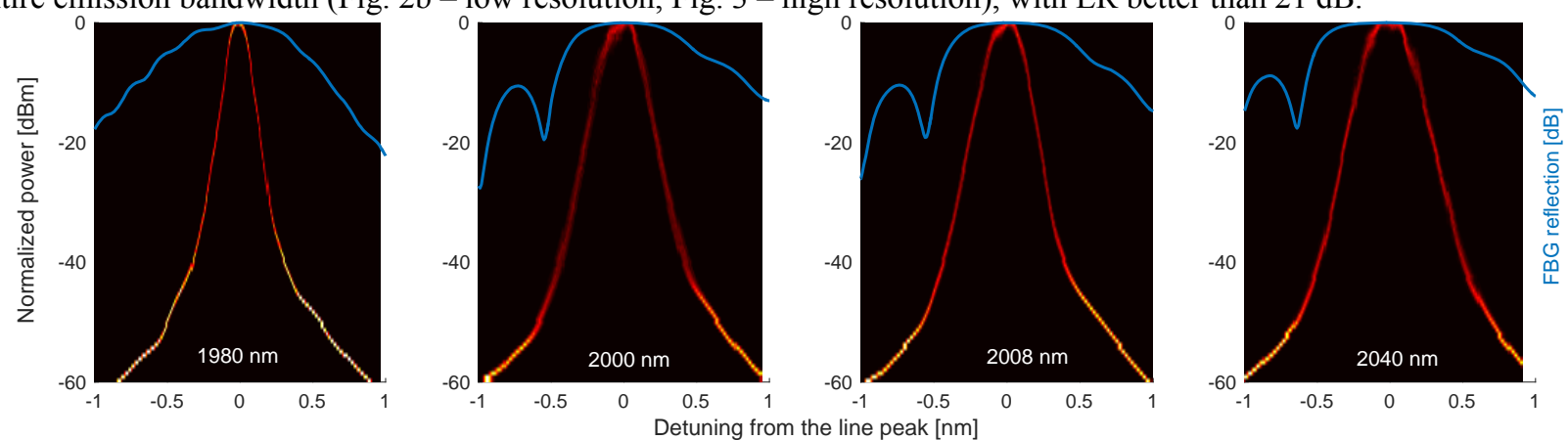

Fig. 3. Laser spectral line shapes for different FBGs, installed in the theta cavity. Every line shape is $2 \mathrm{D}$ histogram, based on 1000 traces. Pump power of $3 \mathrm{~W}, 0.05 \mathrm{~nm}$ resolution. Blue lines - normalized reflection functions of FBGs. 
a)

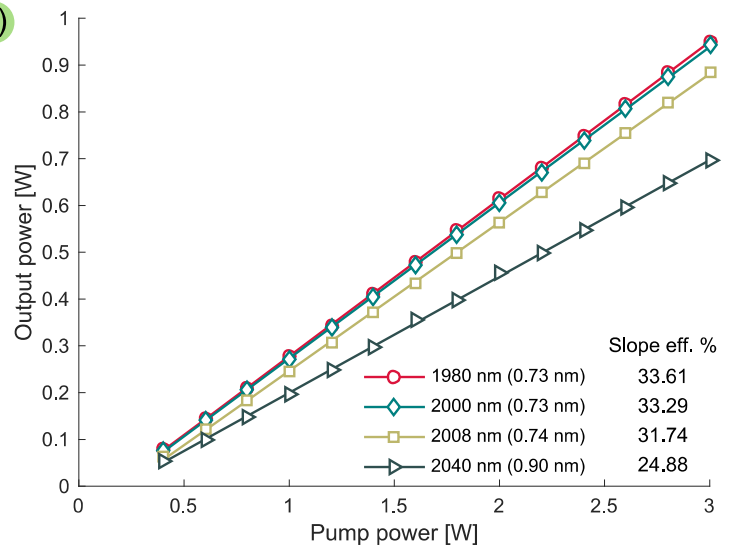

b)

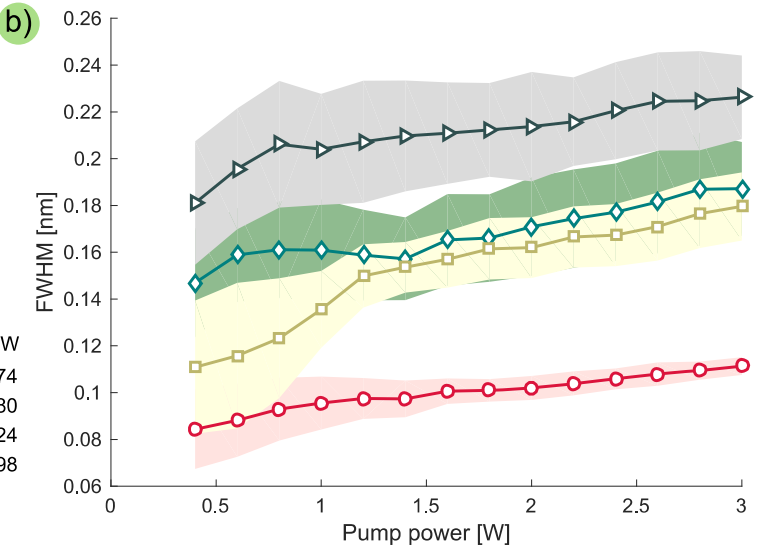

Fig. 4. Laser performance characteristics. A) output power vs. pump power. Inset table shows slope efficiency and pump lasing threshold. B) Laser linewidth (FWHM) vs. pump power. Shadowed regions indicate standard deviation of FWHM (linewidth jitter $\Delta \sigma_{\lambda}$ )

As shown in Fig. 4a, all TDFLs can reach sub-Watt output power level with slope efficiencies of $25-34 \%$ and about $0.2 \mathrm{~W}$ threshold pump power. Overall, the laser performance of theta TDFL with FBGs is improved, comparing to previously reported theta cavity, which exploited a grating-based filter with $4 \mathrm{~dB}$ insertion losses. So, the slope efficiency at $2000 \mathrm{~nm}$ is increased from $25 \%$ (free-space grating) to $33 \%$ (FBG). A minimal slope efficiency of $24.9 \%$ is observed at $2040 \mathrm{~nm}$ due to operation at the edge of TDF gain spectrum. The linewidth at $3 \mathrm{~W}$ pump ranges from $0.1 \mathrm{~nm}$ FWHM at $1980 \mathrm{~nm}$ to $0.22 \mathrm{~nm}$ FWHM at $2040 \mathrm{~nm}$, with a slope of $0.008 \mathrm{~nm} / \mathrm{W}$ for 1980 FBG and $0.013 \mathrm{~nm} / \mathrm{W}$ for other FBGs (Fig. 4b). The FWHM is determined as $2 \sqrt{2 \ln 2} \sigma_{\lambda}$, where $\sigma_{\lambda}$ is the standard deviation of the spectral line profiles in the wavelength domain. Possible fluctuations of FWHM (linewidth jitter $\Delta \sigma_{\lambda}$ ) are shown in Fig. $4 \mathrm{~b}$ as well. The method of evaluation of $\Delta \sigma_{\lambda}$ has been described in the previous publication [11]. Remarkable point is that theta cavity TDFL, operating at $1980 \mathrm{~nm}$, demonstrates a well-stabilized linewidth $\left(\Delta \sigma_{\lambda}\right.$ of $4 \mathrm{pm}$ at $0.1 \mathrm{~nm}$ FWHM). This behavior might be partially attributed to the operation close to the TDF gain peak. However, we believe that the chirping of the FBG strongly contributes to low linewidth jitter, because $2000 \mathrm{~nm}$ laser, exploiting regular FBG and emitting in the vicinity of maximum gain as well, exhibits much higher FWHM deviations $(20 \mathrm{pm})$.

In conclusion, we demonstrate the first truly all-fiber unidirectional TDFL, based on theta cavity configuration with FBG as a filtering element. The laser provides up to $1 \mathrm{~W}$ output power with a slope efficiency of about $30 \%$, preserving the linewidth smaller than $0.22 \mathrm{~nm}$. Using the FBGs with narrower $(\sim 0.1 \mathrm{~nm})$ reflection bandwidth can further reduce the emission linewidth, and improve the laser stability.

This work is supported in part by the Swiss National Science Foundation under grant agreement 200021_140816. We thank the Laboratoire de Physique des Lasers, Atomes et Molécules, Lille University of Science and Technology (France) for the fabrication of 2000, 2008 and $2040 \mathrm{~nm}$ FBGs.

\section{References}

[1] S. Ishii et al, "Coherent $2 \mu \mathrm{m}$ differential absorption and wind lidar with conductively cooled laser and two-axis scanning device," Appl. Opt. 49, 1809-1817 (2010)

[2] S. D. Jackson, "Towards high-power mid-infrared emission from a fibre laser," Nat. Photonics 6, 423-431 (2012).

[3] M. N. Petrovich et al, "Demonstration of amplified data transmission at $2 \mu \mathrm{m}$ in a low-loss wide bandwidth hollow core photonic bandgap fiber," Opt. Express 21, 28559-28569 (2013).

[4] S. Kharitonov at al, "Kerr nonlinearity of Thulium-doped fiber near $2 \mu \mathrm{m}$," in CLEO 2015, p. JTu5A.31.

[5] S. Xing et al, "Characterization and modeling of microstructured chalcogenide fibers for efficient mid-infrared wavelength conversion," Opt. Express 24, 9741-9750 (2016).

[6] Z. Li et al, "All-fiber, ultra-wideband tunable laser at $2 \mu \mathrm{m} .$, " Opt. Lett. 38, 4739-42 (2013).

[7] K. Yin at al, "High-power all-fiber wavelength-tunable thulium doped fiber laser at 2 um," Opt. Express 22, pp. 19947-19952 (2014).

[8] J. Geng et al, "All-fiber wavelength-swept laser near $2 \mu \mathrm{m}$.," Opt. Lett. 36, pp. 3771-3 (2011).

[9] D. Jalas et al, "What is - and what is not - an optical isolator," Nat. Photonics 7, 579-582 (2013).

[10] Y. H. Ja, "Densely spaced two-channel wavelength division demultiplexer with an S-shaped two-coupler optical fiber ring resonator," Appl. Opt. 32, pp. 6679-83 (1993).

[11] S. Kharitonov and C.-S. Brès, "Isolator-free unidirectional thulium-doped fiber laser," Light Sci. Appl. 4, e340 (2015).

[12] M. Chernysheva et al, "High Power Q-Switched Thulium Doped Fibre Laser using Carbon Nanotube Polymer Composite Saturable Absorber," Sci. Rep. 6, p. 24220 (2016). 